\title{
A Novel Approach to Solving Multi-Objective Groundwater Problems with Uncertain Parameters
}

\author{
J.M. Ndambuki* \\ Department of Civil Engineering, Tshwane University of Technology, Private Bag X680, Pretoria, 0001 South Africa
}

\begin{abstract}
As the world population continues to increase, demand for the provision of larger amounts of water to carter for the increased population increases too. Thus, experts in the water resources arena are struggling to meet this everincreasing demand. Moreover, more attention is focusing on groundwater as surface water quality deteriorates. This may lead to conflict unless measures that promote deliberate efficient and sustainable exploitation are embraced. In this paper, we discuss a novel approach to managing groundwater within a multi-objective framework when the input parameters are uncertain. The novelty of the tool is explicitly demonstrated by applying it to an hypothetical example. Results show that the tool is versatile and can prescribe solutions which guarantee desired levels of robustness. While Monte Carlo approach prescribes the use of only 7 wells, the current approach prescribes the use of more wells (up to 16 wells) for a more robust solution. This implies that Monte Carlo approach leads to optimistic solutions which are prone to changes in response to perturbations in input parameters.
\end{abstract}

Keywords: Groundwater, management, uncertainty, second-order cone, monte carlo, multi-objective, decision maker.

\section{INTRODUCTION}

The increasing world population and the need for potable water have seriously stressed the world's water resources. As pollution deteriorates the surface water quality, attention is increasingly being focused on groundwater aquifers. However, over-exploitation of this reserve has adverse impacts, e.g., lowered water table and salt-water intrusion. To avoid such undesirable consequences, it is imperative to understand the behavior of the aquifer when subjected to external stresses. This, coupled with a management scheme will ensure efficient utilization of groundwater resources.

Management of groundwater resources can be done either deterministically or stochastically. In a deterministic approach, it is assumed that all the input data is known without error while stochastic approach recognises the fact that uncertainty in input data is real, hence should be addressed. Deterministic methods, therefore, require, full characterization of aquifers which is neither practical nor economically feasible, hence different approaches are required which are capable of utilizing the available scanty data so as to design strategies which recognizes the uncertainty brought about by inadequacy of data. This recognition has led to stochastic methods which treat the inadequate input data as stochastic processes, thus one has to solve a stochastic optimization problem. Furthermore, when the problem of scanty input data is compounded with the desire by a decision maker (DM) to consider more than one objective, the available solution methods of stochastic optimization which presupposes that the DM's preferences are conveniently

\footnotetext{
*Address correspondence to this author at the Department of Civil Engineering, Tshwane University of Technology, Private Bag X680, Pretoria, 0001 South Africa; Tel: +27(0)123825225; Fax: +27(0)123825226;

E-mail: jmndambuki@yahoo.co.uk
}

rendered through a single objective are no longer applicable. Our contribution in this paper is to:

- Present a combined simulation and optimization methodology which considers the uncertainty brought about by the lack of adequate data to fully characterize the groundwater aquifer

- Recognizes that in a real-world situation, it is often the case that a DM's preferences are articulated through more than one objective (implying a multiobjective optimization approach).

Since groundwater management is generally carried out in an environment of uncertainties, the question of reliability of the model output is of paramount importance. Heterogeneity in natural aquifer formations is widely recognized as one of the major factors contributing to uncertainty in predicting groundwater flow behavior and management strategies. In an attempt to incorporate uncertainty in groundwater management, a number of methodologies have been developed and published in the literature. They include post-optimality analysis (e.g., [1, 2]) chance-constrained programming and stochastic optimization with recourse method as presented by [3-6].

In this paper, we transform our stochastic groundwater quantity management problem into a second-order cone optimization (SOCO) problem, which is then solved by a powerful interior-point method. We first present a general introduction to SOCO problems followed by the formulation of a second-order cone groundwater quantity management problem. Subsequently, we present results for an example which is solved using this tool.

Consider a linear optimization problem (LOP) of the following form: 
$\operatorname{minimize} c^{T} x$

subject to:

$a_{i}^{T} x \leq b_{i}, \quad i=1, \ldots, m$,

$x \geq 0$,

where:

$c, a_{i} \in R^{n} ; b_{i} \in R$ are the problem parameters while $x$ are the optimisation variables. Assuming that all the problem parameters except $a_{i}$ are accurately known and that $a_{i}$ are uncertain but lying in ellipsoids $\varepsilon_{\mathrm{i}}$ defined as:

$a_{i} \in \varepsilon_{i}=\left\{\bar{a}_{i}+\mathbf{P}_{i} u_{i} \mid\left\|u_{i}\right\| \leq 1\right\}$,

where:

$\mathbf{P}=\mathbf{P}^{\mathrm{T}}$ are $n \times n$ perturbation matrices; $\overline{a_{i}}$ are the nominal values and the norm of $u_{i}$ ensure convexity, then a robust solution of the optimization problem given by Eqs. (1) to (3) is as follows:

minimize $c^{T} x$

Subject to:

$a_{i}^{T} x \leq b_{i}, \quad \forall a_{i} \in \varepsilon_{i}, \quad i=1, \ldots, m$,

$x \geq 0$.

An optimization problem defined by Eqs. (5) to (7), though now deterministic, has infinitely many constraints and a solution to this robust optimization problem is feasible if for all $i=1, \ldots m$, the following holds:

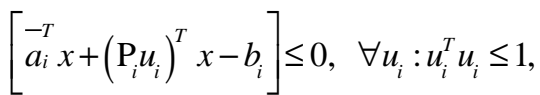

which can equivalently be reformulated by a single constraint as:

$\bar{a}_{i}^{T} x+\left\|\mathrm{P}_{i} x\right\| \leq b_{i}$

Constraints of the form Eq. (9) are referred to as secondorder cone constraints (otherwise known as Lorentz cone or ice-cream cone constraints). Thus the optimization problem defined by (1)-(3) can explicitly be written as a SOCO problem as follows:

minimize $c^{T} x$

Subject to:

$-T$
$a_{i} x+\left\|\mathrm{P}_{i} x\right\| \leq b_{i}, \quad i=1, \ldots, m$.

The norm term is the usual Euclidean norm and can be thought of as a penalty term which introduces some robustness within the optimization problem.

In multi-objective optimization, one is interested in optimizing (minimizing or maximizing) several objectives simultaneously. The consideration of several objectives simultaneously leads to an optimization approach known as multiobjective or vector optimization. This approach recognizes the fact that not all the objectives can achieve their optimal values simultaneously unless the objectives are not competing (conflicting). This means that there is no unique solution to such problems. However one may establish a specific numeric goal (also known as aspiration level) for each of the objectives and then seek a solution that minimizes the sum of deviations of the objective functions from their respective goals. This solution process is known as goal programming.

Consider now the $\mathrm{n}$-dimensional vector space $\mathbf{R}^{\mathrm{n}}$. For any two points $\mathbf{r}=\left(r_{1}, \ldots, r_{n}\right)$ and $\bar{r}=\left(\bar{r}_{1}, \ldots, \bar{r}_{n}\right)$, we can express the distance $d(r ; \bar{r})$ as the norm given by $\|d\|=\|r-\bar{r}\|$. Thus the distance between the two points can be measured by the $L_{p}$ - metric (Holder's norm) as:

$\|d\|_{p}=\left(\sum_{0=1}^{k}\left|r_{k}-\bar{r}_{k}\right|^{p}\right)^{\frac{1}{p}}$

Let $\bar{Z}=\left\{\bar{Z}_{1}, \ldots \bar{Z}_{K}\right\}^{T}$ be the aspiration levels (values of objectives which the decision maker wishes to achieve on the various objectives) and $Z(x)=\left\{Z_{1}(x), \ldots, Z_{k}(x)\right\}^{T}$ be the objectives the decision maker is considering. The distance between vectors $\bar{Z}$ and $\mathrm{Z}(\mathrm{x})$ can be measured by the Holder's norm as:

$\|\bar{Z}-Z(x)\|_{p}=\left(\sum_{k=1}^{k}\left|\bar{Z}_{k}-Z_{k}(x)\right|^{p}\right)^{\frac{1}{p}}$

To avoid biased solution and express decision maker's (DM's) preferences towards the considered objectives, weighting and w can be applied to Eq. (13) which then becomes

$\| \bar{Z}-Z(x)) \|_{w_{1} p}=\left(\sum_{k-1}^{k} w_{k}\left|\bar{Z}_{k}-Z_{k}(x)\right|^{p}\right)^{\frac{1}{p}}$

By using the $L_{2}$ metric, one would then solve an optimization problem of the following form in order to minimize the distance between $\bar{Z}$ and $\mathrm{Z}(\mathrm{x})$ within the feasible set, $\Omega$.

$\operatorname{minimize}\|\bar{Z}-Z(x)\|_{w, 2}$

Where $\mathrm{x}$ is the optimization variable and the other parameters are as defined before. By introducing a scalar deviational variable $\delta$, the Eq. (15) translates to the following:

minimize $\delta$

Subject to:

$\|\bar{Z}-Z(x)\|_{w, 2} \leq \delta$

Which a SOCO problem as long is as $\Omega$ is defined by linear or second-order cone constraints. Note that inequality (17) implies that $\delta \geq 0$.

Having presented a general introduction on multiobjective optimization in the preceding sub-section, we now present a formulation of the multi-objectives groundwater 
management problem, which we seek to solve. In this problem, one of our objectives is to minimize the operational cost while the other objective is to maximize the amount of water extracted from the groundwater aquifer. Clearly, the two objectives are not only conflicting (increasing the amount of water extracted would necessarily result in an increase in operational cost) but also non-commensurate (different units in the objective attributes) and hence the complexity involved in the solution process. Our multi-objective SOCO problem is as follows:

$$
\begin{aligned}
& \operatorname{minimize}\left[Z_{1}(x)=c^{T} x\right], \\
& \operatorname{maximize}\left[Z_{2}(x)=e^{T} x\right],
\end{aligned}
$$

Subject to:

$$
\begin{aligned}
& \bar{a}_{i}^{T} x+\left\|P_{i}^{T} x\right\| \leq b_{i}, \quad \mathrm{i}=1, \ldots, \mathrm{N}_{\mathrm{c}}, \\
& e^{T} x \geq W_{d}, \\
& 0 \leq x_{j} \leq U_{j}, j=1, \ldots, N_{w},
\end{aligned}
$$

Where $c=\left(c_{1} \ldots \ldots, c_{j} \ldots \ldots, c_{N w}\right)^{T}$,

$a_{i}=\left(a_{i, 1} \ldots, a_{i j} \ldots a_{i, N W}\right)^{T}, \mathrm{e}=\left(1_{1}, . ., 1_{\mathrm{j}}, \ldots, 1_{\mathrm{NW}}\right)^{\mathrm{T}}$,

$x=\left(x_{i},,, x_{j}, \ldots, x_{N w}\right)^{T}$ and

- $\mathrm{N}_{\mathrm{w}}$ is the number of pumping wells

- $\mathrm{N}_{\mathrm{c}}$ is the number of control points

- $\mathrm{c}_{1}$ is the aggregated daily cost of pumping and transportation in monetary units (MUs) per unit volume at cell $\mathrm{j}$;

- $\mathrm{x}_{\mathrm{j}}$ is the pumping rate in cell $\mathrm{j}$;

- $\mathrm{a}_{\mathrm{IJ}}$ is the response at control point $\mathrm{i}$ due to pumping in cell $\mathrm{j}$;

- $b_{I}$ is the constraining value at control point $\mathrm{i}$;

- $\mathrm{W}_{\mathrm{d}}$ is total water demand;

- $\mathrm{U}_{\mathrm{j}}$ is the maximum pumping rate allowed in cell $\mathrm{j}$.

By introducing a deviational variable, $\delta$ and considering the $L_{2}$ metric (Euclideen distance) as the measure of closeness between the aspiration levels $\bar{Z}_{1}$ and $\vec{Z}_{2}$ and the feasible objective region, the above multi-objective optimization problem (Eq. (18)-(22)) can be reformulated as:

$\operatorname{minimize} \delta$,

subject to:

$$
\begin{aligned}
& \|\bar{Z}-Z(x)\|_{w, 2} \leq \delta, \\
& \bar{a}_{i}^{T} x+\left\|P_{i}^{T} x\right\| \leq b_{i}, \quad i=1, \ldots, N_{c}, \\
& e^{T} x \geq W_{d}, \\
& 0 \leq x j \leq U_{j}, j=1, \ldots, N_{w},
\end{aligned}
$$

Where $\delta$ is a scalar variable, $\bar{Z}=\left(\bar{Z}_{1}, \bar{Z}_{2}\right)^{T}$ are the aspiration levels of objectives $Z(x)=\left(Z_{1}(x) ; Z_{2}(x)\right)^{T}$ and objectives $Z(x)$ are as defined by Eq. (18) and (19) respectively. The other parameters and variables are as defined before. To express DM preferences, preference values can be included in inequality (24) when such preferences exist.

SOCO problems can be solved efficiently through the interior-point methods that have been developed ([7-9]). A few applications of SOCO problems have been reported in the literature. They include antenna array weight design, filter design, grasping force optimization, portfolio optimization, truss design, and equilibrium of systems with piecewiselinear springs design. From the literature, the reported applications are basically in the areas of electrical engineering, mechanical engineering, economics, and structural engineering $[5,8,10-13]$.

\section{Application of the Methodology to an Example}

We use a hypothetical example to demonstrate the applicability of the methodology outlined above. Our goal in this problem is to supply domestic water to a distribution centre situated in the middle of a single confined square aquifer of thickness $35 \mathrm{~m}$. The aquifer is an island of dimensions $30 \mathrm{~km}$ and with parameters as shown in Figs. 1 and 2.

\section{Objective:}

The objective is to supply the required amount of water at the lowest possible cost while at the same time satisfying the hard (deterministic) constraints and ensuring robustness of the optimal solution.

Constraints: DM expresses constraints as follows:

- In the specified ecological protect zone, the minimum water level equals $5 \mathrm{~m}$ above sea level.

- The hydraulic head in all the nodes except those in contact with the sea are bounded by the bottom of the aquifer

- To avoid saltwater intrusion, head in cells next to the sea are not allowed to fall below $0.2 \mathrm{~m}$ above sea level.

- There is minimum water demand of $3 \mathrm{~m}^{3} / \mathrm{s}$ at the distribution centre which should be satisfied.

- Pumping rates from potential wells shown in Fig. 3 are limited to a maximum yield of $1.5 \mathrm{~m}^{3} / \mathrm{s}$

- Unit costs of exploitation measured in monetary units (MUs) defined at each potential well are calculated as a combination of the water pumping costs and water transport costs which depend on the distance from the well to the distribution centre. The costs are assumed to be higher towards the boundaries. The aggregated unit cost coefficients at each cell take on values from $0.1 \mathrm{MU}$ at the centre and increase at a rate of $0.1 \mathrm{MU}$ for every $200 \mathrm{~m}$ distance. Note that MU can be in any currency units.

The problem is to find the location of wells and the corresponding pumping rates, satisfying all or nearly all the constraints on the hydraulic heads and pumping rates and the minimum demand at the distribution centre. Moreover, the 


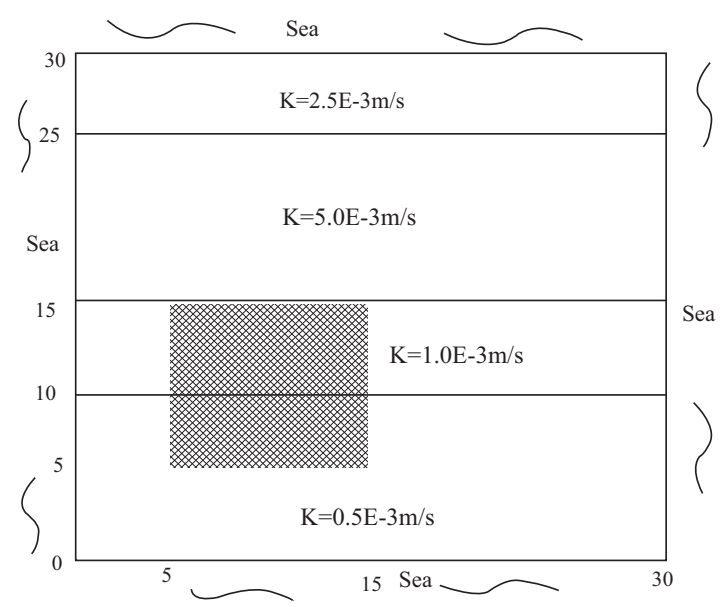

Fig. (1). Ecological protection zone and conductivity zones.

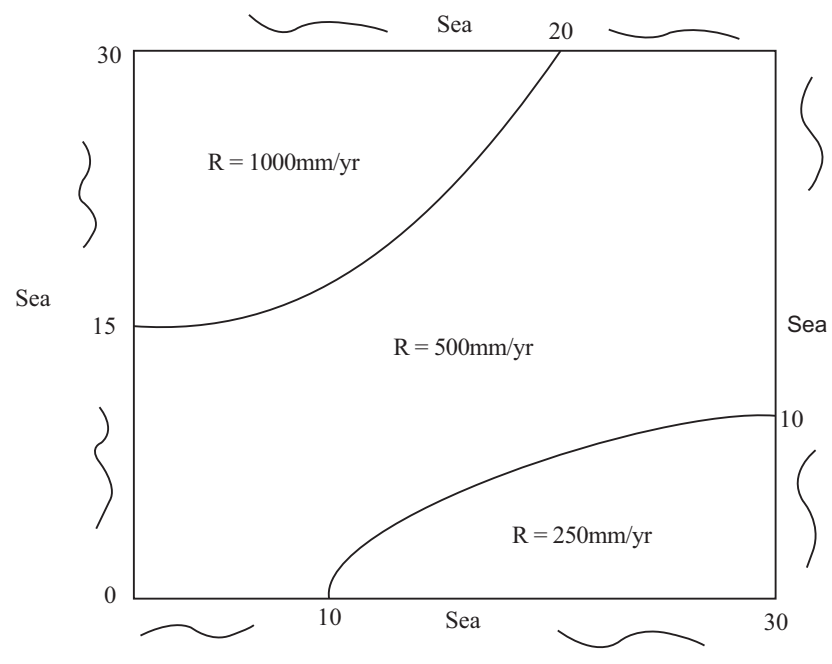

Fig. (2). Recharge zones.

solution should be robust in an environment of uncertain spatial hydraulic conductivity values.

\section{DISCUSSION OF RESULTS}

For the analysis of the example we have outlined, 20 realizations of the uncertain hydraulic conductivity, k; were generated using the zonal $\mathrm{k}$ values shown in Fig. 1, a standard deviation $(\log )$ of 0.5 and a correlation length of 30 $000 \mathrm{~m}$ in $\mathrm{x}$-direction and $7500 \mathrm{~m}$ in $\mathrm{y}$-direction. These correlation lengths were chosen so as to replicate the hydraulic conductivity field of the example as used by [2] with a view to comparing the results. All the SOCO problems were solved using Sturm's SeDuMi package [9].

The multi-objective SOCO problem solved (Eqs. 23 to 27) resulted in 50 decision variables (49 of them dealing with the location of pumping wells and their strengths and 1 variable measuring the deviation of the optimal solution from the aspiration levels), 101 linear deterministic constraints, 1 deterministic second-order cone constraint to minimise the deviation from the aspiration levels and 225 second-order cone constraints to capture the robustness (coefficients of these constraints are uncertain, hence stochastic). The targets (aspiration levels) for the two objective functions were computed as $\overline{\mathrm{Z}}_{1}=2.07 \mathrm{MU}$ and $\overline{\mathrm{Z}}_{2}=4.47 \mathrm{~m}^{3} / \mathrm{s}$.

We then solved the multi-objective optimisation problem defined by Eqs. 23 to 27 for various levels of robustness. As in the case of the single objective SOCO problem already discussed, a scaling factor $\eta$ can be introduced in inequality (25) by replacing the perturbation matrices $P_{i}$ by $\eta P_{i}$ where $\eta \geq 0$. If $\eta=0$, it means that there is no uncertainty (the input parameters are exactly known), $\eta=1$ means that the uncertainty is given by the matrices $P_{i}^{T} P_{i}$ and $\eta>1$ it means that the uncertainty is higher than that given by the covariance matrices $P_{i}^{T} P_{i}$.

Table 1 shows the levels of robustness and the corresponding number of active wells and values of the operational cost (these results are for a guaranteed volume of water amounting to $3.0 \mathrm{~m}^{3} / \mathrm{s}$ ). The results show that as the level of robustness $\eta$ is increased from 0 to 0.3 , the number of active wells increases and consequently the operational cost. This is because increasing the level of robustness implies increase in the volume of the ellipsoid and hence one has to search for a solution within a zone of increasingly high uncertainty. The consequence is that each active well will pump less and since the guaranteed volume of water must be realized, then more pumping wells will have to be mobilized resulting to higher operational costs.

Table 2 and Fig. 4 shows how the deviational variable, $\delta$, varies with the level of robustness (remember that the deviational variable is a measure of the discrepancy between the aspiration level, in this case the ideal vector, and the optimal or compromise solution realized). It is interesting to note that as the level of robustness is increased, the devia-

\begin{tabular}{|l|l|l|l|l|l|l|}
\hline P1 & P2 & P3 & P4 & P5 & P6 & P13 \\
\hline P8 & P9 & P10 & P11 & P12 & P14 & P20 \\
\hline P15 & P16 & P17 & P18 & P21 & P27 & P28 \\
\hline P22 & P23 & P24 & P25 & P33 & P34 & P35 \\
\hline P29 & P30 & P31 & P38 & P39 & P40 & P42 \\
\hline P43 & P37 & P45 & P46 & P47 & P48 & P49 \\
\hline
\end{tabular}

Fig. (3). Location of potential pumping wells. 


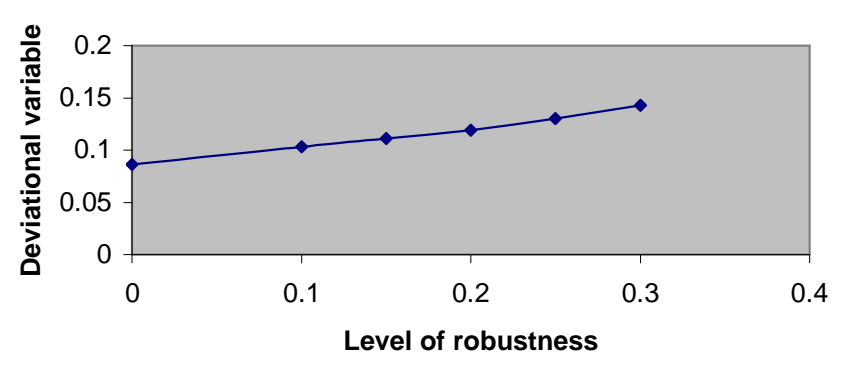

Fig. (4). Robustness vs. deviational variable.

Table 1. Robustness vs. Active Wells a Cost

\begin{tabular}{|c|c|c|}
\hline Level of Robustness & Active Wells & Cost (MU) \\
\hline \hline 0.0 & 11 & 2.160 \\
\hline 0.1 & 14 & 2.176 \\
\hline 0.15 & 14 & 2.185 \\
\hline 0.2 & 16 & 2.191 \\
\hline 0.25 & 19 & 2.193 \\
\hline 0.3 & 19 & 2.195 \\
\hline
\end{tabular}

Table 2. Deviational Variable vs. Robustness

\begin{tabular}{|c|c|}
\hline Level of Robustness & Deviational Variable, \\
\hline \hline 0.0 & 0.086 \\
\hline 0.1 & 0.103 \\
\hline 0.15 & 0.111 \\
\hline 0.2 & 0.119 \\
\hline 0.25 & 0.130 \\
\hline 0.3 & 0.143 \\
\hline
\end{tabular}

tional variable increases too. This is because an increase in robustness means an increase in the volume of the ellipsoid which implies that the solution sought must guard against a wider range of uncertainty, hence more conservative. The consequence is that such a solution will lie somewhere in the interior of the feasible set. Thus, as the level of robustness is increased further, the solutions will come from increasingly deeper into the feasible set.

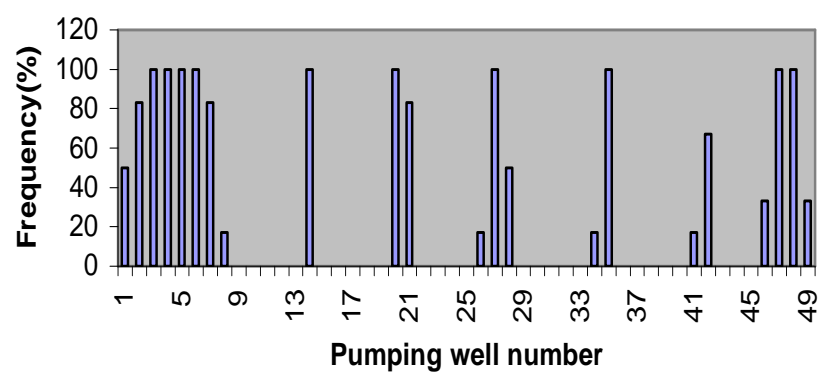

Fig. (5). Well use frequency.

From Table 1, consider the 3 levels of robustness, namely $0.0,0.2$, and 0.3 . These levels of robustness were chosen so as to show how the optimal solutions evolve as the levels of robustness are increased. From the results, pumping wells $\mathrm{p}_{3}$; $\mathrm{p}_{4} ; \mathrm{p}_{5}, \mathrm{p}_{6} ; \mathrm{p}_{14} ; \mathrm{p}_{20} ; \mathrm{p}_{27}, \mathrm{p}_{35}, \mathrm{p}_{47}$ and $\mathrm{p}_{48}$ are used $100 \%$ of the cases; $\mathrm{p}_{2} ; \mathrm{p}_{7} ;$ and $\mathrm{p}_{21} ; 83 \% ; \mathrm{p}_{42} ; 67 \% ; \mathrm{p}_{1}$ and $\mathrm{p}_{28} ; 50 \% ; \mathrm{p}_{46}$ and $\mathrm{p}_{49} ; 33 \%$; while $\mathrm{p}_{8} ; \mathrm{p}_{26} ; \mathrm{p}_{34}$ and $\mathrm{p}_{41}$ are used $17 \%$ of the cases. The rest of the pumping wells are not used at all. Fig. 5 shows how frequently each of the wells is used. This figure (compare with Fig. 3 which shows the location of pumping wells) shows that pumping wells located to the north and east of the model domain are used more often than pumping wells located elsewhere as expected (these pumping wells are located away from the ecological protection zone).

Compromise solutions corresponding to various quantities of water extracted are given in Table 3 (these solutions are for 0.2 level of robustness). It is apparent that as the quantity of water extracted is increased, the operational cost likewise increases. This depicts some trade-off between the two conflicting and non-commensurate objectives. The optimal schemes corresponding to the optimal solutions Numbers 1, 3, and 5, are depicted in Figs. (6-8). Similarly, these optimal solutions are chosen so as to show how the pumping strategies evolve under different objective trade-offs.

The results indicate that as the water demand is increased from a minimum of $3.0 \mathrm{~m}^{3} / \mathrm{s}$ to a maximum of $3.45 \mathrm{~m}^{3} / \mathrm{s}$, the operational cost increases from a minimum of $2.191 \mathrm{MU}$ to a maximum of $2.670 \mathrm{MU}$. The number of active pumping wells range from a minimum of 14 wells to a maximum of 20 wells. Fig. (9) gives an indication of how frequently each pumping well is used across the 5 optimal trade-off solutions given in Table 3 .

[13] compared the optimal solutions for the Monte Carlo approach (which they referred to as stochastic scenario) and the SOCO approach (which they referred to as robust scenario). Their results showed that the robust scenario gives rise to a more expensive optimal strategy. This is because the

Table 3. Volume vs. Cost

\begin{tabular}{|c|c|c|}
\hline Optimal Solution Number & Volume $\left(\mathbf{m}^{3} / \mathbf{s}\right)$ & Cost $(\mathbf{M U})$ \\
\hline \hline 1 & 3.0 & 2.191 \\
\hline 2 & 3.15 & 2.234 \\
\hline 3 & 3.25 & 2.348 \\
\hline 4 & 3.3 & 2.419 \\
\hline 5 & 3.45 & 2.670 \\
\hline
\end{tabular}




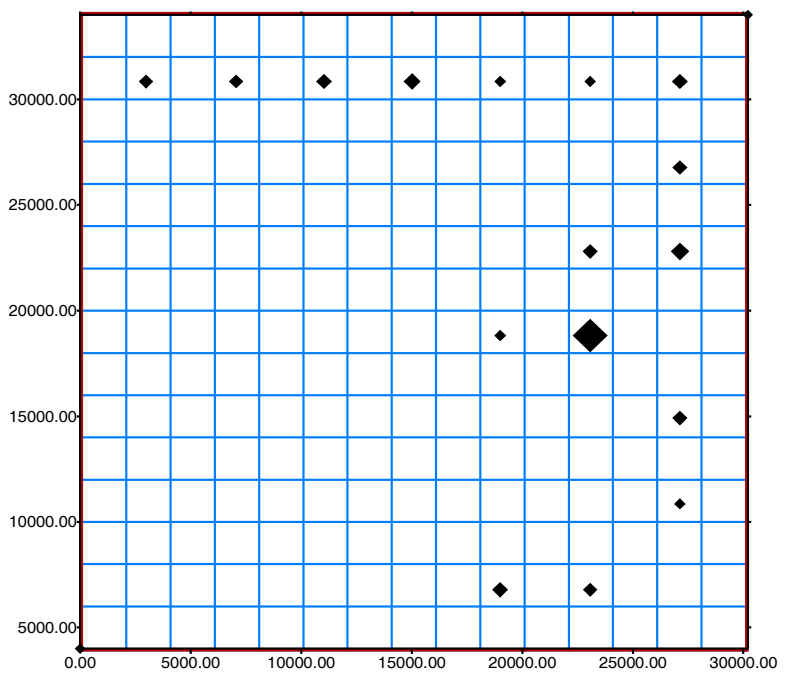

Fig. (6). Optimal solution Number 1.

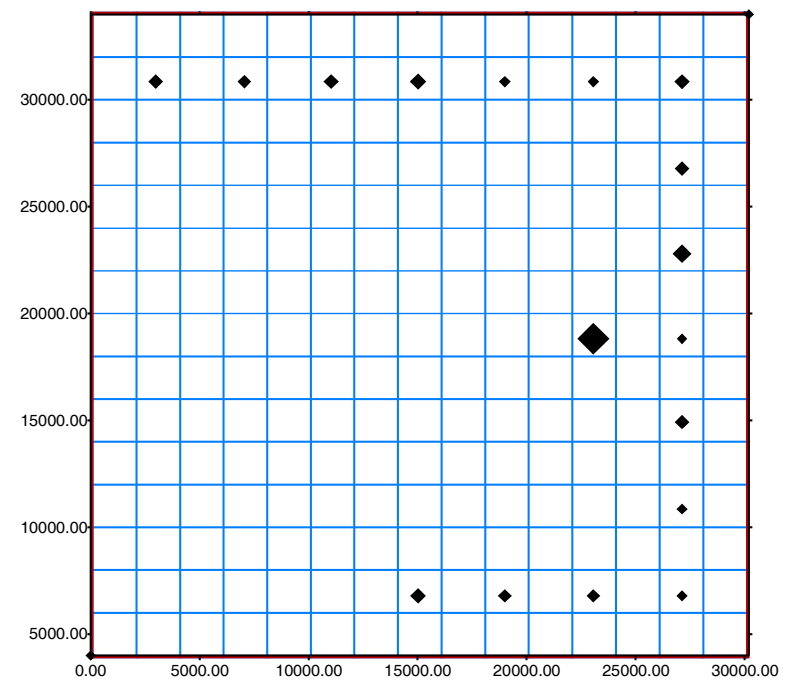

Fig. (7). Optimal solution Number 3.

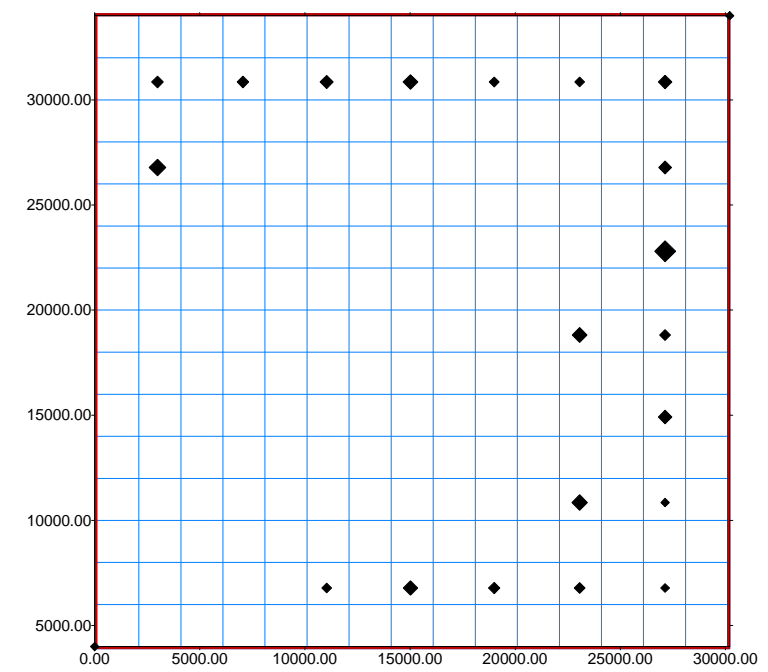

Fig. (8). Optimal solution Number 5.

robust optimal strategy operates more wells (16 wells) compared to the Monte Carlo optimal strategy which operates 7 wells. This implies that Monte Carlo approach gives rise to optimistic solutions compared to SOCO approach. The

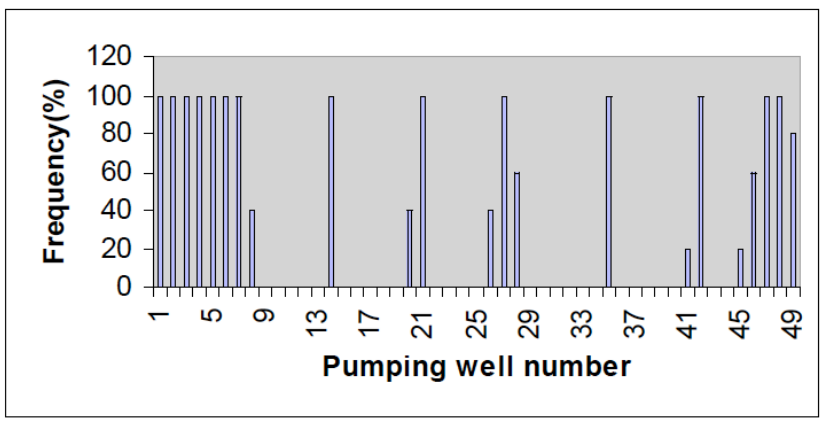

Fig. (9). Well - use frequency for trade-off solutions.

authors further performed a post-optimality sensitivity analysis to evaluate how the two optimal solutions would perform in an environment of uncertainty. The results showed that SOCO approach gives rise to more stable solutions than the Monte Carlo approach (total constraint violations are less than those of the Monte Carlo approach solution). For detailed comparison of the performance of the optimal solutions of the two approaches, see [3].

\section{CONCLUSIONS}

In this paper, we have shown that when confronted with uncertain multi-objective linear optimization problems, such problems can be cast as SOCO problems which are efficiently solved by the interior point methods. We have further demonstrated, through an example, how one could apply this novel tool to efficiently manage water resources in an environment of uncertainty. Using this methodology, one can easily increase or decrease the robustness of the solutions and therefore be able to choose an optimal strategy taking into account the values of the objectives considered and the level of robustness such a solution is able of guarantee. An advantage of this approach is that one does not have to consider a large number of realizations to derive reasonable statistics of the uncertain parameters (as is the case with the Monte Carlo approach). Moreover, by approaching the uncertainty problem through $\mathrm{SOCO}$, one is assured of robust solutions that are unlikely to be adversely affected by minimal perturbations within the problem parameters. By recognizing that every system is fraught with uncertainty, it is the desire of every DM to be presented with such robust solutions.

\section{REFERENCES}

[1] Aguado E, Sitar N, Remson I. Sensitivity analysis in aquifer studies. Water Resour Res 1977; 13(4): 733-7.

[2] Gorelick SM. A model for managing sources of groundwater pollution. Water Resour Res 1982; 18(4): 773-81.

[3] Ndambuki JM. Multi-Objective groundwater quantity management: A stochastic approach. Delft University Press 2001.

[4] Wagner JM, Shamir U, Nemati HR. Groundwater quality management under uncertainty: Stochastic programming approaches and the value of information. Water Resour Res 1992;28(5): 1233-46.

[5] Ndambuki JM, Otieno FAO, Stoet CBM, Veling EJM. Groundwater management under uncertainty: A multi-objective approach. Water SA 2000; 26(1): 35-42.

[6] Mulvey JM, Vanderbei RJ, Zenios SA. Robust optimization of large-scale systems. Oper Res 1995; 43(2): 264-81.

[7] Andersen ED, Andersen KD. Exploiting parallel hardware to solve optimization problems. SIAM News 1999; 32(4): 1-6.

[8] Boyd S, Vandenberghe L, Grant M. Efficient convex optimization for engineering design. In: Proc. IFAC Symposium on robust control design, 1994. Rio-de-Janeiro, Brazil. 
[9] Sturm JF. Using SeDuMi 1.02, A Matlab toolbox for optimization over symmetric cones. In: Potra F, Roos C, Terlaky T, Eds. Optimization methods and software 1999; Vol. 11-12: pp. 625-654 (Special Issue on Interior Point Methods).

[10] Lobo MS, Vandenberghe L, Boyd S, Lebret H. Applications of second-order cone programming. Linear Algebra Appl 1998; 193228.

[11] Ben-Tal A, Nemirovski A. Convex optimization in engineering 1998; Technion-Israel Institute of Technology.
[12]

Boyd S, Crusius G, Hansson A. Control applications of non-linear convex programming. J Process Control 1998; 8(5-6): 313-24.

[13] Ndambuki JM, Stoet CBM, Veling EJM, Terlaky T. Robust Groundwater management through second order cone programming (SOCP). In: Oliver Sililo et al. Ed. Proceedings of the $\mathrm{XXX}^{\text {th }}$ IAH congress, Groundwater: Past Achievements and Future Challenges 2000: pp. 413-7.

Received: April 12, 2010

Revised: September 29, 2010

Accepted: November 26, 2010

() J.M. Ndambuki; Licensee Bentham Open.

This is an open access article licensed under the terms of the Creative Commons Attribution Non-Commercial License (http://creativecommons.org/licenses/by-nc/3.0/) which permits unrestricted, non-commercial use, distribution and reproduction in any medium, provided the work is properly cited. 